\title{
The influence of the no symmetric stress tensor on the flow separation
}

\author{
EVELINA PROZOROVA \\ Mathematical-Mechanical Department \\ St. Petersburg State University \\ Av. 28 , Peterhof, 198504, RUSSIA
}

\begin{abstract}
The aim of this work is to investigate the influence of the angular momentum in aerodynamic for problem of flow separation and study some the numerical schemes that we can use to solute received equations. We received non- symmetric tensor from investigation of continuous mechanics and from kinetic theory. In a modified theory, the laws of conservation in integral form remain the same, but the divergent and rotational components are not distinguished. The correspondence between the potential flow with velocity circulation, Newton law and the equations by Euler is discussed for investigation problem of flow separation. The analysis of the influence of the moment on the separation of the flow is carried out. Some numerical schemes which can be used to calculate modified equations, based on the Runge-Kutta method for approximating an time's operator are analyzed.
\end{abstract}

Keywords:: angular moment, potential flow, circulation, Euler equation, Runge-Kutta method

Received: November 2, 2019. Revised: May 8, 2020. Accepted: May 17, 2020.

Published: May 19, 2020.

\section{Introduction.}

There are many experimental facts that currently cannot be described theoretically. A possible reason is bad mathematical models and algorithms for calculation, despite the many works in this area of research. There are problems, for example, in the theory of elasticity, which cannot be solved using the symmetric stress tensor. [1]. The starting point of a computational experiment is the choice of a mathematical model. Models are selected that can be investigated using mathematical tools at their disposal. The number of methods that used in physics to study mathematical models is not enough: dimensional analysis of the problem, asymptotic methods for large (small) distances or times, for large (small) values of some dimensionless parameter of the problem; reducing the dimension of the problem by averaging the equations; methods of separation of variables in its various variations, the linearization of the problem, etc. These methods are applicable for a limited class of tasks, as a rule, for linear problems under certain conditions. The equations of mathematical physics express the laws of conservation (mass, momentum, energy, angular momentum, charge, etc.). Since there are not so many laws, there are few equations. They express the laws of conservation in each specific science. In mathematical modeling, we use either the integral formulation corresponding to the experimental data, or the differential formulation following from it when setting an additional condition for the smoothness of the functions. Therefore, when passing to the differential formulation, the problems with discontinuity do not fall into the domain of definition, that is tasks with discontinuity surfaces such as shock waves, contact surface problems and. etc. In classical mechanics, in addition, when using the Ostrogradsky Gauss theorem discarding the out of integral item means ignoring the velocity circulation over the surface of the selected volume. When taking into account the motion of a gas, this term is difficult to introduce into the differential equation. We can receive equations from physical and geometric suggests.

Therefore, to account for all components of the motion, it is proposed to use an integral formulation. So we obtain modified the Navier-Stokes and Boltzmann equations. Thus, we have non symmetric tensor from 
investigation of continuous mechanics and from kinetic theory.

The non-symmetric tensor is obtained, therefore, when the velocity circulation is taken into account, i.e. when accounting for the rotation of an elementary volume. The only mechanism leading to rotation is the action of the moment of force. This question was considered in $[2,3]$.

Back in 1970, in the textbook [4] it was said, "First of all, we note that along with the distribution of volume and surface forces, for the sake of generality, we should also consider the volume and surface distributions of pairs of forces (moments), on the possibility of which in

continuous media is currently indicated". A.A. Ilyushin. pointed out the role of the moment [5]. He drew attention to the fact that in the classical theory, matter "breaks". In our work the issues discussed concern structureless particles. For structural particles, the Cosserat theory [6] is widely used. The rotation of the elementary volume during decomposition of speed in a row should occur relative to the center of inertia, but not relative to an arbitrary axis; the contribution to the conservation laws for an arbitrarily located volume relative to the coordinate system is provided not only by normal but also by tangent components; the center of inertia of a set of molecules moves in accordance with the distribution of velocities; ignoring outside the integral term is only suitable for a potential flow without twist. Molecular motion is always there if the temperature is not zero (Kelvin). Therefore, even for one-dimensional movement will be observed additional effects in the form of self-diffusion and thermal diffusion. This effect creates additional item in the equations and is observed, for example, during the formation of a direct shock wave. [7,8].

«For large Reynolds numbers in the lowfrequency and inertial intervals of turbulent motion, the influence of molecular viscosity and small flow elements in most of the disturbance region on the general characteristics of the macrostructures of the developed flow and on the flow pattern as a whole is practically insignificant, which makes it possible to ignore the effects of molecular viscosity when studying the dynamics of large vortices and study them on the basis of models of an ideal medium, for example, Euler equations. " [9]. For research it was proposed to use integral laws. For a compressible gas, the implementation of a numerical solution is difficult due to the need to take into account changes in the elementary volume.

When considering a potential flow, it is possible to compare the influence of velocity circulation to the pressure gradient in the Euler equations. This will reveal that part of the speed disappears in the Euler equations. In fact, not taking into account the full speed and transferring to the nonequilibrium process the conclusions and experimental data for equilibrium states, we cannot obtain experimental results of theory. "The simplest physical of flow separation models assume two-dimensionality of the flow, i.e., its independence from one of the spatial coordinates (in this case, along the leading edge of the wing). Recent studies have shown the limitations of this idealization: when turbulent separation and flow separation from the leading edge in the separation zone, large-scale vortices are formed along the wing span, which are associated with intense gas movement in the transverse direction. The existence of such vortices fundamentally changes the physical picture of the flow compared with that considered in two-dimensional problems of flow separation.'[10]. In a modified theory, the laws of conservation in integral form remain the same, but the divergent and rotational components are not distinguished. The experiments also do not distinguish the individual components of the velocities, including when determining the viscosity coefficient. Of course, the physical meaning of each term is important, and at small speeds of motion the classical theory works well, although it contains assumptions that do not correspond to classical theoretical mechanics and classical mathematics, since it was formulated at the time of low speeds. We may assume that the following provisions were ignored: the rotation of the elementary volume that was received after 
the velocity was putting in a row should occur relative to the center of inertia, but not relative to an arbitrary axis; the contribution to the conservation laws for an arbitrarily located volume relative to the coordinate system is provided not only by normal but also by tangent components; the center of inertia of a set of molecules moves in accordance with the distribution of velocities; ignoring outside the integral term is only suitable for a potential flow. Molecular motion is always there if the temperature is not zero (Kelvin).

The mathematical model of a physical phenomenon includes, in addition to the basic equations expressing the general laws of conservation, some additional relations describing the properties of specific media and which are actually coefficients of equations. These are the coefficients of thermal conductivity, diffusion, electrical conductivity, radiation absorption, viscosity, etc. These relations include the equations of state. All relationships are functions of the environment. Since the resulting equations in most cases cannot be solved analytically, they are solved numerically, i.e. we replace the continuous medium with a discrete one. Besides model we need to have method for solving these equations. At present, the most promising numerical methods are considered to be the Runge-Kutta methods of increased order of accuracy, based on the use of the finite volume method with approximation of derivatives with respect to the coordinate with a high order of accuracy. A characteristic feature of the method is the calculation without intermediate time points, although the classical method uses values at intermediate time points. A formal analysis shows that in this case the circuit remains a second-order approximation circuit, but the stability of the circuit increases. More reasonable is the factorized scheme proposed in the work. However, the task is rarely reduced to the form indicated in the work. The construction of the finite volume method is based on classical differential equations by averaging, i.e. full conservation laws are not implemented. The very method of constructing conservation laws takes into account incomplete derivatives of velocities, creating a problem, for example, to compute the separation of the boundary layer. In the experiments, the divergent and rotor parts of the velocities are not distinguished. Transfer coefficients are determined, as well as equations of state, from experiments. Therefore, Newton's law for stresses must contain the full speed. It should be used in the Navier-Stokes equations without decomposition of speed in a row. The existing classical theory is constructed so that the main role is played by pressure forces. This is probably why, at low speeds, results coincidences with experiment are obtained. However, calculations even for a potential flow lead to the formation of a vortex sheet. For Euler's equations, this means that Pascal's law in the nonequilibrium case does not work and it is necessary to consider separately not pressure $p$, but $p_{x}, p_{y}, p_{z}$. An example of the importance of separating the individual components is the study of the processes of wave propagation in a rotating fluid [11] with the introduction of a item related to rotation. in pressure gradient. The used values of the functions in the nodes of the difference schemes, no matter how we reduce the step, give asymmetric pressure values. In previous works, numerical examples of solving the simplest problems of the theory of elasticity, a boundary layer, and kinetic theory were given [8]. The inclusion of velocity circulation in potential flows means the inclusion of rotational velocity components.

The work discusses the listed issues and, if possible, gives answers to some of them. The main attention is paid to constructing a mathematical model for calculating the problem of flow separation from the surface.

\section{The influence of the angular momentum in the equations of aerodynamics.}

There are problems that cannot be solved within the framework of the symmetric stress tensor. For example, (Fig.1) [1] 


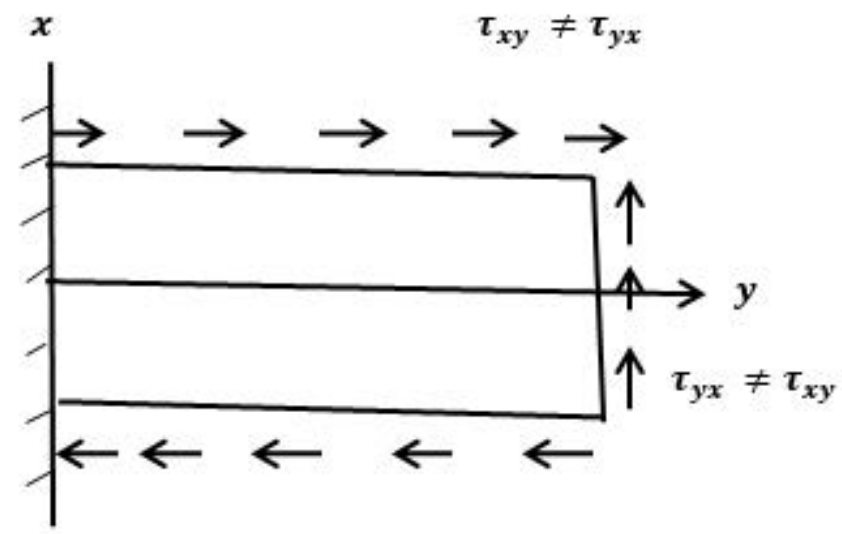

Fig. 1. An example of the inapplicability of a symmetric tensor

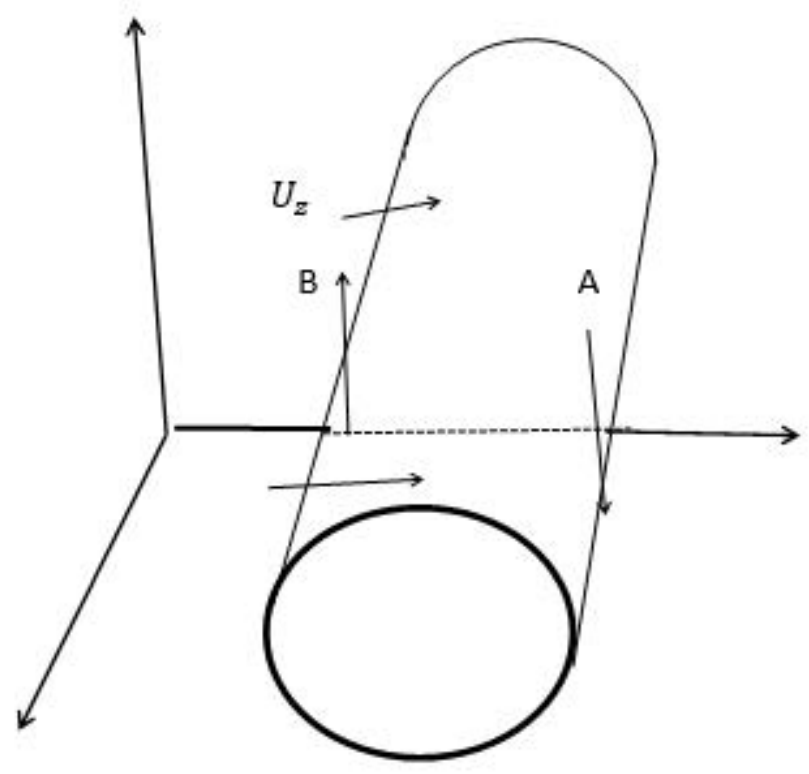

Fig. 2 Formation of circulation rate

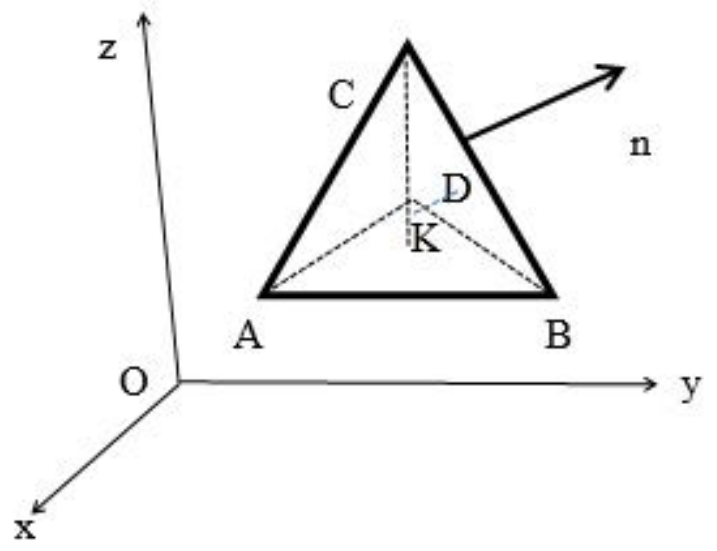

Fig3 Pressure determination 
The angular momentum is three-dimensional in nature and the vortex is formed if an angular momentum.is applied. For poorly streamlined bodies near the separation point, the role of the moment increases due to a change in the sign of the derivative in the pressure distribution or due to deceleration of the flow to zero speed. In calculations of an inviscous gas, a vortex sheet appears, but the pressure $P_{x}, P_{y}, P_{z}$ at the boundary, for example, the wings from below and above and on the side surfaces changes not only as a result of the action of these components, but also as a result of the force generated by the moment. Infinitely thin wings all the same have lateral surfaces, air moves, giving a force that creates transverse eddies. The parameters of the vortices depend on the thickness of the profile and the magnitude of the flow velocity.

The side vortices are almost entirely determined by the moments. When elucidating the causes of flow separation from the wings during their flow around and analyzing the results of calculations using the Euler and Navier-Stokes models, it should be taken into account that the kinetic theory proves the first order of approximation of viscous terms. When obtaining conservation laws from kinetic theory and further designing on coordinate axes, it is possible to distinguish pressure components along the selected axis. The basic order leads to the Euler equations, and the calculation according to these equations leads to the appearance of a vortex sheet. Therefore, the classical theory of choosing the representation of an elementary volume by a point and in computational methods with consideration of finite volumes must be determined differently.

But in classical mechanics

$-P_{n}=-\alpha P_{x},-P_{n}=-\beta P_{y},-P_{n}-\gamma P_{z}$. So

$$
P_{n}=P_{x}=P_{y}=P_{z}=p
$$

In all textbooks, the physical cause of circulation is associated with the presence of viscosity in the fluid. It seems likely that in addition to the indicated reason, a moment may arise as a result of the pressure gradient. For example, under equilibrium conditions in a potential field of volume forces for an incompressible fluid, the projections of moments are determined by

$$
\begin{gathered}
M_{x}=y p_{3}-z p_{1}, \quad M_{Y}=-x p_{3}+z p_{1}, \\
M_{z}=x p_{2}-y p_{1} .
\end{gathered}
$$

An additional force arises in $\mathrm{x}$ and in $\mathrm{z} F_{x}=$ $-\rho g x, F_{y}=-\rho g z$. Only with such a distribution of stresses is a condition of equilibrium possible without the movement of a liquid or gas. When flowing around a thin flat plate, separation should not occur. It occurs only when the plate moves at an angle of attack. Usually they are only interested in the position of the center of application of the moment, but the distributed moments, creating additional force, should influence the position of the flow separation point without influence of the viscosity.

Regarding a thin plate, the following can be said: When solving the problem of flow around a plate on the basis of Euler equations, the pressure distribution along the surface is asymmetric due to conditions of no flowing on normal to the surface, and this fact must be taken into account in a moment. Really

$$
\left[\begin{array}{ccc}
i & j & k \\
x & y & z \\
p_{1} & p_{2} & p_{3}
\end{array}\right]
$$

The following fact can serve as a proof: for potential flows, the Euler equations written in terms of the potential function $\varphi$ and the stream function $\Psi$ look as

$$
\begin{aligned}
& u \frac{\partial u}{\partial x}+v \frac{\partial u}{\partial y}=-\frac{\partial p}{\partial x} \Rightarrow \frac{\partial \varphi}{\partial x} \frac{\partial^{2} \varphi}{\partial x^{2}}+\frac{\partial \varphi}{\partial y} \frac{\partial^{2} \varphi}{\partial y \partial x} \\
& =-\frac{\partial p}{\partial x} \\
& u \frac{\partial v}{\partial x}+v \frac{\partial v}{\partial y}=-\frac{\partial p}{\partial y} \Rightarrow \frac{\partial \varphi}{\partial x} \frac{\partial^{2} \varphi}{\partial y \partial x} \\
& +\frac{\partial \varphi}{\partial y} \frac{\partial^{2} \varphi}{\partial y^{2}}=-\frac{\partial p}{\partial y}
\end{aligned}
$$

Express in terms of the stream function $\psi$

$\frac{\partial \psi}{\partial y} \frac{\partial^{2} \psi}{\partial x^{2}}-\frac{\partial \psi}{\partial x} \frac{\partial^{2} \Psi}{\partial y \partial x}=-\frac{\partial p}{\partial y}$ 
The equations are different and the boundary conditions are different. Therefore, different values of the pressure will be obtained in the $x$ and $y$ coordinates.

Then, even in flat problems, pressure of the third coordinate begins to affect . Therefore, the determination of large-scale vortices according to the Euler equations for an ideal gas is close to their distribution obtained from the Navier-Stokes equations.

In the work [ ] the mathematical models of describing for rarefied gas and continuous mechanics and to study the errors that arise when we describe a rarefied gas through distribution function. Writing physical values conservation laws via delta functions, the same classical definition of physical values are obtained as in classical mechanics. Kinetic theory makes it impossible to consider these effects, since the molecules do not differ in the distribution function (on for pressure and on for force of momemtum). In numerical calculations in aerodynamics, it is not customary to average values over the volume. Continuity of the medium is achieved by adopting the asymmetry of the stress tensor. Euler equations have a pressure gradient, but the results obtained by Pascal, for the equilibrium case, are transferred to the nonequilibrium case. It should be noted that the derivation of the Boltzmann equation itself corresponds to a first-order approximation scheme. In this case, the question arises whether it is necessary to increase the accuracy of solving the equation.

In classical mechanics, the equations for gas and liquid have the form () with the hypothesis about the symmetry of the stress tensor.

$$
\begin{aligned}
& \rho\left(\frac{\partial u}{\partial t}+u \frac{\partial u}{\partial x}+v \frac{\partial u}{\partial y}+w \frac{\partial u}{\partial z}\right)=\rho F_{x}+\frac{\partial P_{x x}}{\partial x} \\
& +\frac{\partial P_{y x}}{\partial y}+\frac{\partial P_{z x}}{\partial z} \\
& \rho\left(\frac{\partial v}{\partial t}+u \frac{\partial v}{\partial x}+v \quad \frac{\partial v}{\partial y}+w \frac{\partial v}{\partial z}\right)=\rho F_{x} \\
& \frac{\partial P_{x y}}{\partial x}+\frac{\partial P_{y y}}{\partial y}+\frac{\partial P_{z y}}{\partial z} \\
& \text { (2.9) } \\
& \rho\left(\frac{\partial w}{\partial t}+u \frac{\partial w}{\partial x}+v \quad \frac{\partial w}{\partial y}+w \frac{\partial w}{\partial z}\right)=\rho F_{z} \\
& \frac{\partial P_{x z}}{\partial x}+\frac{\partial P_{y z}}{\partial y}+\frac{\partial P_{z z}}{\partial z}
\end{aligned}
$$

$$
\begin{gathered}
y\left(\frac{\partial \sigma_{x z}}{\partial x}+\frac{\partial \sigma_{y z}}{\partial y}+\frac{\partial \sigma_{z z}}{\partial z}\right)-z\left(\frac{\partial \sigma_{x y}}{\partial x}+\right. \\
\left.\frac{\partial \sigma_{y y}}{\partial y}+\frac{\partial \sigma_{z y}}{\partial z}\right)+\sigma_{z y}-\sigma_{z y}=0 \\
x\left(\frac{\partial \sigma_{x y}}{\partial x}+\frac{\partial \sigma_{y y}}{\partial y}+\frac{\partial \sigma_{z y}}{\partial z}\right)-y\left(\frac{\partial \sigma_{x x}}{\partial x}+\right. \\
\left.\frac{\partial \sigma_{y x}}{\partial y}+\frac{\partial \sigma_{z x}}{\partial z}\right)+\sigma_{y x}-\sigma_{x y}=0 \\
x\left(\frac{\partial \sigma_{x z}}{\partial x}+\frac{\partial \sigma_{y z}}{\partial y}+\frac{\partial \sigma_{z z}}{\partial z}\right)-z\left(\frac{\partial \sigma_{x x}}{\partial x}+\right. \\
\left.\frac{\partial \sigma_{y x}}{\partial y}+\frac{\partial \sigma_{z x}}{\partial z}\right)+\sigma_{z x}-\sigma_{x z}=0
\end{gathered}
$$

Then we highlight the viscous pressure item and the addend that is associated with pressure in an ideal gas. In the modified theory, the viscous component and the pressure gradient are still distinguished. However, if we consider the pressure equal to one third of the sum of the pressures on the coordinate platforms, then the gas inside the volume should not change its speed. These are physical principles. If the gradient works, then equality is impossible.

Consequently, each coordinate will have its own pressure, and instead of one $\mathrm{p}$ value, take the values $p_{x}, p_{y}, p_{z}$ It is required to take into account the influence of the distributed moment of force. Its influence is local, the value depends on the gradients of physical quantities.

In previous works, the reasons for the appearance of the angular momentum in the Navier-Stokes equations were clarified. The role of the nonintegral term was established with allowance for the rotation of an elementary volume in the Ostrogradsky Gauss theorem. An algorithm was proposed for determining the degree of asymmetry of the viscous stress tensor and the order of the terms of this tensor was clarified. It is important that the equation of state contains the total speed including the rotational component. Newton's law is carried out in accordance with experimental data. Full speed is known

$V_{k t}^{\prime}=V+\frac{1}{2} \operatorname{rot} V$ 


$$
\begin{aligned}
& u_{k T}^{\prime}=u+\frac{1}{2}\left(\frac{\partial u}{\partial z}-\frac{\partial w}{\partial x}\right)\left(z-z^{\prime}\right)- \\
& \frac{1}{2}\left(\frac{\partial v}{\partial x}-\frac{\partial u}{\partial y}\right)\left(y^{\prime}-y\right), \\
& v_{k T}^{\prime}=v+\frac{1}{2}\left(\frac{\partial v}{\partial x}-\frac{\partial u}{\partial y}\right)\left(x^{\prime}-x\right)- \\
& \frac{1}{2}\left(\frac{\partial w}{\partial y}-\frac{\partial v}{\partial z}\right)\left(z^{\prime}-\mathrm{z}\right), \\
& w_{k T}^{\prime}=w+\frac{1}{2}\left(\frac{\partial w}{\partial y}-\frac{\partial v}{\partial z}\right)\left(y^{\prime}-y\right) \\
& -\frac{1}{2}\left(\frac{\partial w}{\partial y}-\frac{\partial v}{\partial z}\right)\left(x^{\prime}-x\right) . \\
& \frac{d u^{\prime}}{d t}=\frac{1}{2} \frac{d r}{d t} \times \operatorname{rot} V+\frac{1}{2}\left(\mathrm{r}-r^{\prime}\right) \times \frac{d}{d t} \operatorname{rot} w
\end{aligned}
$$

If we focus on the above, we can assume that in the viscous case only translational speed will be important.

\section{About some difference schemes}

An important role, in addition to choosing a model, is the choice of a solution method. When solving problems of aeromechanics, the control volume method is often used at present by using for approcsimation on time Runge-Kutta schemes with various modifications. The finite volume method is an integral method. If it comes from the initial experimental setting, then it grasps all the components involved in the change in value physical values in the volume. However, now the method is being built on the basis of existing differential equations. In addition, the method uses the values of functions on time layers without passing to intermediate points in time. Intermediate values are replaced by the introduction of additional coefficients. We give two versions of schemes that, after excluding intermediate calculations, do not lead to the Taylor series. Schemes are three-layer on time, explicit. The latter gives them an advantage over implicit ones. The latter gives them an advantage over implicit ones, but it is not to get a higher order than traditional explicitly implicit ones.

First, consider the classic version of the method

$$
y^{\prime}=f(x, y), \quad y\left(x_{0}\right)=y_{0}
$$

$$
\begin{aligned}
& {\left[x_{0}, X\right], \quad X>x_{0} \text {. }} \\
& x_{1}=x_{0}+h<X, h>0 . k_{1}, k_{2}, k_{3}, k_{4} \\
& k_{1}=h f\left(x_{0}, y_{0}\right) \text {, } \\
& k_{2}=h f\left(x_{0}+\frac{h}{2}, \quad y_{0 .}+\frac{k_{1}}{2}\right) \\
& k_{3}=h f\left(x_{0}+\frac{h}{2}\right. \text {, } \\
& y_{0} \\
& \left.+\frac{k_{2}}{2}\right) \\
& k_{4}=h f\left(x_{0}+h, \quad y_{0 .}+k_{3}\right) \\
& \begin{aligned}
y_{1}=y_{0 .} & +\left(\frac{1}{6}\right)\left(k_{1}+2 k_{2}+2 k_{3}\right. \\
& \left.+k_{4}\right)
\end{aligned}
\end{aligned}
$$

In the work, the implementation of the method is as follows

$$
\begin{aligned}
& Q_{i, j, k}^{(1)}=Q_{i, j, k}^{(0)}+\Delta t L\left(Q_{i, j, k}^{(0)}\right) ; \\
&\left.Q_{i, j, k}^{(2)}=\frac{3}{4} Q_{i, j, k}^{(0)}+\frac{1}{4}\left[Q_{i, j, k}^{(1)}+\Delta t L\left(Q_{i, j, k}^{(1)}\right)\right] ;\right) \\
&\left.Q_{i, j, k}^{(2)}=\frac{1}{3} Q_{i, j, k}^{(0)}+\frac{2}{3}\left[Q_{i, j, k}^{(2)}+\Delta t L\left(Q_{i, j, k}^{(2)}\right)\right] ;\right) \\
& y^{(1)=y^{(0)}}+\Delta t f\left(x_{0}, y_{0 .}\right), \\
& y^{(2)=\frac{3}{4} y^{(0)}}+\frac{1}{4}\left[y^{(0)}+\Delta t f\left(x_{0}, y_{0 .}\right)\right. \\
&+\Delta t f\left(y^{(0)}+\Delta t f\left(x_{0}, y_{0 .}\right)\right] \\
& y^{(3)=\frac{1}{3} y^{(0)}}+\frac{2}{3}\left\{\frac{3}{4} y^{(0)}+\frac{1}{4}\left[y^{(0)}\right.\right. \\
&+\Delta t f\left(x_{0}, y_{0 .}\right) \\
&+\Delta t f\left(y^{(0)}+\Delta t f\left(x_{0}, y_{0 .}\right)\right] \\
&+\Delta t f\left\{\frac{3}{4} y^{(0)}+\frac{1}{4}\left[y^{(0)}\right.\right. \\
&+\Delta t f\left(x_{0}, y_{0 .}\right) \\
&+\Delta t f\left(y^{(0)}\right. \\
&\left.\left.\left.+\Delta t f\left(x_{0}, y_{0 .}\right)\right]\right\}\right\}
\end{aligned}
$$

However, after expanding into a series and performing the summation, we have

$y^{(1)}=y^{(0)}+\Delta t{\frac{d y^{(0)}}{d t}}^{(\cdots}+\cdots$

The following term is equal to the coefficient $1 / 2$ times $f\left(x_{0}, y_{0}\right)$ Therefore, we do not get the Taylor series.

Therefore, we do not get the Taylor series.

It follows that the circuit has a second order of accuracy, but its stability increases. In schemes [13,14], the approximation order does not coincide with the declared one. 
Scheme [15] of the third and fourth orders in time and space. Implicit.

The factorization method. In orthogonal curvilinear coordinates

$$
\begin{aligned}
& \operatorname{div} \mathbf{A}=\frac{1}{H_{1} H_{2} H_{3}}\left[\frac{\partial\left(H_{2} H_{3} A_{q_{1}}\right)}{\partial q_{1}}+\frac{\partial\left(H_{3} H_{1} A_{q_{2}}\right)}{\partial q_{2}}+\right. \\
& \left.\frac{\partial\left(H_{1} H_{2} A_{q_{1}}\right)}{\partial q_{3}}\right], \text { but no } \\
& \frac{\partial U}{\partial t}+\frac{\partial F}{\partial \xi}+\frac{\partial G}{\partial \eta}+\frac{\partial H}{\partial \zeta}=\frac{\partial F_{v}}{\partial \xi}+\frac{\partial G_{v}}{\partial \eta}+\frac{\partial H_{V}}{\partial \zeta} \\
& \zeta, \xi, \eta \text {-functions } x, y, z
\end{aligned}
$$

The transition to Cartesian coordinates will be reflected in the order of errors. What boundary conditions can be used for this scheme is not clear. Factorization is difficult .. In addition. Excludedваться для данной схемы, не ясно.

$$
\begin{aligned}
& (\boldsymbol{V} \cdot \nabla) \boldsymbol{V}=\operatorname{rot} \boldsymbol{V} \times \boldsymbol{V}+\operatorname{grad}\left(\frac{V^{2}}{2}\right) \\
& {[(\boldsymbol{V} \cdot \nabla) \boldsymbol{V}]_{i}=[\operatorname{Div}(\boldsymbol{V} \boldsymbol{V})-\boldsymbol{V} \operatorname{div} \boldsymbol{V}]_{i}}
\end{aligned}
$$

The inviscid part of the operators and the viscous are separately factorized. Curvilinear coordinate system without source, matrices are reduced to diagonal form. The scheme maintains the declared order for the divergent form of equations, which is not always possible. Some numerical schemes which can be used to calculate modified equations, based on the Runge-Kutta method for approximating an time's operator are analyzed.

\section{Conclusion}

Analysis of classical mathematical models of continuum mechanics and influence of the angular momentum on problem separation flow are discussed. A new mathematical model is proposed that takes into account the non-integral term that is responsible for the influence of the action of the angular momentum and includes the equation of state. The non-integral term is related to the circulation of velocity over the volume, i.e. with the moment. Differential equations constructed from geometric and physical data that include these components are proposed. . The analysis of the influence of the moment on the separation of the flow is carried out. The correlation of equations in potential functions and Euler equations indicates that the pressure does not coincide for different directions. Some numerical schemes which can be used to calculate modified equations, based on the RungeKutta method for approximating an time's operator are analyzed.

\section{References}

[1] E. A. Bulanov. Moment stresses in the mechanics of a solid, bulk and liquid body. M, University book. 2012.140s. [2] E. V. Prozorova. The Effect of Angular Momentum and Ostrogradsky-Gauss Theorem in the Equations of Mechanics. Wseas Transaction on fluid Mechanics DOI: 10.37394/232013.2020.15.2

[3].Evelina V. Prozorova. Effect of Mathematical Models on Experimental Data for the Gas and Liquids. Journal of Mechanics Engineering and Automation 6 (2016) 313-318

doi: $10.17265 / 2159-5275 / 2016.06 .008$

[4] L.G.Loytsyansky. Fluid Mechanics. M.: Nauka.1970.904c.

[5] A.A. Ilyushin. Asymmetry of strain and stress tensors in the mechanics of a continuous medium. WEST. Moscow. University. Ser. 1. Mathematics-mechanics. ! 996. p. 6-14

[6] A.Ye. Ishlinskii, D.D. Ivlev. The mathematical theory of plasticity. M.: Fizmatlit, 2003, 702s. [7]. S.V. Vallander, M.P. Elovskih. The theoretical dependence of the heat conductivity of gases on temperature. Reports of the USSR Academy of Sciences, 1951, V. $79 \quad$ (1). 37-40. [8]. Evelina Prozorova. Influence of the dispersion in model of continuous mechanics. Lambert. 2018, 108

[9].O.M. Belotserkovsky, A.M. Oparin, V.M. Chechetkin. Turbulence: new approaches. M .: Science. 2003.286

[10]. V.V. Kozlov. Physics of flow structures/ Flow separation. https://vihrestruktura.3dn.ru/publ/2-1-0-6.

Novosibirsk State University 
[11].S.V. Alekseenko, P.A. Kuybin, V.L. Okulov. Introduction to the theory of concentrated vortices. Moscow-Izhevsk. Institute for Computer Research. $2005.504 \mathrm{~s}$. [12]. S.R. Chakravarthy, S. Osher. A new class of hugh-accuracy TVD schemes for hyperbolic conservation ; laws AIAA Paper. N/ 85-0363 1985

[13]. Antony Jameson, Wolfgang Schmidt, Eli Turkel Numerical Solution of the Euler Equations by Finite Volume Methods Using Runge-Kutta Time-Stepping Schemes AIAA Paper 1981.1259

[14]. Pawel Buchm•ul leryand Christiane Helzelz. Improved Accuracy of High-Order WENO Finite Volume Methods on Cartesian Grids_ Department of Mathematics, Ruhr-University Bochum January 29, 2014

[15]. V.G. Croup. 3rd order numerical method for time integration of the NavierStokes equations. Journal of Computational Mathematics and Mathematical Physics. T. 59, N. 11, 2019 p. 1948-1960 